\title{
RANDOM ERRORS OF TYCHO ASTROMETRY ${ }^{1}$
}

\author{
V.V. MAKAROV AND E. HØG \\ Copenhagen University Observatory - NBIfAFG \\ Østervoldgade 3, DK-1350 Copenhagen K, Denmark
}

\begin{abstract}
The paper focuses on the problem of estimation of Tycho astrometry random errors, caused by photon noise. A theoretical model for the random errors was developed on the basis of the Maximum Likelihood estimator. The model provides a satisfactory agreement with experimental data in the wide range of star magnitudes from 4 to 10 for both vertical and inclined slit transits. It is confirmed theoretically, that for fainter stars, which constitute a half of the Tycho star sample, this model is not valid. Moreover, it is demonstrated that a reliable astrometry could hardly be achieved for these faint stars, were it not for a kind of Bayesian approach which is in fact implemented in the processing. This approach uses implicitly a priori astrometric information on positions of the stars. A major drawback of the method is that it introduces some bias in astrometry estimation, of presently unknown size. Nonetheless, no transfer of systematic errors from the input catalogue is expected, for the used prior information comes from Recognition, hence it is based purely on the satellite's own observations. - The inadequacy of the pure ML theory leads us to correct the model empirically, in order to provide reliable formal errors for the astrometric parameters in the final catalogue, for all Tycho magnitudes. In this way, a rms normalized residual is used for each star individually as a scale factor or correction to the formal covariances. Corrected in this way, formal errors are compared directly with external errors, calculated from absolute differences between the Tycho provisional parallaxes in a 30 months solution and the Hipparcos parallaxes of some 100000 common stars. Analysis of a standard external error shows that the corrected formal errors are robust, and probably even overestimated.
\end{abstract}

\footnotetext{
${ }^{1}$ Based on observations made with the ESA Hipparcos satellite
} 